\title{
Repetition blindness is immune to the central bottleneck
}

\author{
Paul E. Dux and René Marois \\ Vanderbilt University, Nashville, Tennessee
}

\begin{abstract}
The attentional blink (AB) and repetition blindness (RB) phenomena refer to subjects' impaired ability to detect the second of two different $(\mathrm{AB})$ or identical $(\mathrm{RB})$ target stimuli in a rapid serial visual presentation stream if they appear within $500 \mathrm{msec}$ of one another. Despite the fact that the $\mathrm{AB}$ reveals a failure of conscious visual perception, it is at least partly due to limitations at central stages of information processing. Do all attentional limits to conscious perception have their locus at this central bottleneck? To address this question, here we investigated whether RB is affected by online response selection, a cognitive operation that requires central processing. The results indicate that, unlike the $\mathrm{AB}, \mathrm{RB}$ does not result from central resource limitations. Evidently, temporal attentional limits to conscious perception can occur at multiple stages of information processing.
\end{abstract}

Despite the impressive processing power of the human brain, it is severely limited in capacity. For example, we can barely attend to more than a few objects at once, and we can barely perform two tasks simultaneously (see Marois \& Ivanoff, 2005, for a recent review). Such processing limitations are readily observed at both perceptual and response stages of information processing. Two phenomena that illustrate severe capacity limitations in conscious perception are the attentional blink (AB; Chun \& Potter, 1995; Raymond, Shapiro, \& Arnell, 1992; Sergent \& Dehaene, 2004) and repetition blindness (RB; Johnston, Hochhaus, \& Ruthruff, 2002; Kanwisher, 1987; Morris \& Harris, 2004). The AB refers to subjects' impaired ability in perceiving the second (T2) of two distinct targets in a rapid serial visual presentation (RSVP) stream of distractor stimuli, if the second target appears within 200-600 msec of the first target (T1). RB is similar to the $\mathrm{AB}$, in that it, too, reveals impairment in target recognition under dual-task conditions; however, it differs from the AB in that it specifically refers to subjects' impaired ability to report both occurrences of a repeated target stimulus in an RSVP stream. Despite the fact that the $\mathrm{AB}$ and $\mathrm{RB}$ are phenomenologically similar, these two deficits can be experimentally dissociated. Chun (1997) demonstrated that, whereas the $\mathrm{AB}$ was abolished when letter targets appeared amongst keyboard symbol distractors, RB was still present. Conversely, whereas RB was eliminated when targets differed in color from one another and the distractors, a robust $\mathrm{AB}$ was still elicited under these conditions (see Ward, Duncan, \& Shapiro, 1997, for a further dissociation between $\mathrm{RB}$ and the $\mathrm{AB}$ ). On the basis of these findings, Chun (1997; see also Ruthruff \& Pashler, 2001) suggested that the two phenomena resulted from distinct capacity-limited processes; the $\mathrm{AB}$, he hy- pothesized, reflected a failure in encoding target identity information into working memory, whereas RB was a result of a subject's inability to individuate two identical stimuli as distinct perceptual events during encoding.

A capacity limitation that has its locus at responserelated stages of information processing is the psychological refractory period (PRP; Telford, 1931). The PRP effect is elicited when subjects are required to make speeded responses to two stimuli. While reaction time to the first stimulus is typically unaffected by manipulations of stimulus onset asynchrony (SOA), performance on the second task becomes increasingly slowed as SOA decreases. This effect is thought to result from a central processing bottleneck occurring at the stage of response selection (Pashler, 1994; Pashler \& Johnston, 1989), although other cognitive processes, such as memory retrieval (Carrier \& Pashler, 1995) and mental rotation (Ruthruff, Miller, \& Lachmann, 1995), may also require the same central resources.

The PRP differs from the AB and RB paradigms in two important ways. First, while the PRP paradigm requires online responses to both targets, responses are typically made after the end of the RSVP stream in AB/RB paradigms. Second, while the perceptual system is strained by presenting stimuli successively at a rapid pace in RSVP paradigms, the slower, distractor-free presentation of stimuli in the PRP task does not create data limitations at the perceptual level. Because $\mathrm{AB} / \mathrm{RB}$ paradigms appear to tax the perceptual system, whereas the PRP task places a greater burden on the response end of the information pathway, these paradigms have been considered to reveal distinct processing limitations (Pashler, 1998). However, studies using hybrid AB-PRP paradigms have demonstrated that the $\mathrm{AB}$ and PRP partly tap into a common processing bottleneck (Arnell \& Duncan, 2002; 
Jolicœur, Dell' Acqua, \& Crebolder, 2001; Ruthruff \& Pashler, 2001). For example, speeding the response to $\mathrm{T} 1$ increases the magnitude of the AB (Jolicœur, 1998; Ruthruff \& Pashler, 2001). Conversely, simply making an unspeeded perceptual decision on $\mathrm{T} 1$ is sufficient to trigger a PRP for T2 (Jolicœur et al., 2001; Ruthruff \& Pashler, 2001). These findings have led to the suggestion that the encoding of information into working memory may not proceed concurrently with online response selection (Jolicœur, 1998; Jolicœur et al., 2001). While such studies have yet to fully elucidate the cognitive process(es) common to both the AB and PRP (Jolicœur et al., 2001; Luck \& Vogel, 2001; Marois \& Ivanoff, 2005; Ruthruff \& Pashler, 2001), they nonetheless clearly establish that conscious target recognition and response selection tap into a common central bottleneck of information processing.

To what extent is this central bottleneck limiting what we see and do at any given moment? Specifically, do limitations in central processing resources underlie all forms of temporal limits to attentive perception? To address these questions, we investigated whether RB, also, has its locus at the central bottleneck. In the following experiment, we manipulated whether the first letter target in a hybrid ABRB paradigm required a speeded or a delayed response. Online response selection in unpracticed, arbitrary sensorimotor tasks is a tried-and-true diagnostic test for the involvement of the central bottleneck of information processing (Pashler, 1998). If RB magnitude is increased in speeded trials relative to unspeeded trials, this would provide evidence that the individuation of target events draws on the same capacity-limited central resources as does the encoding of the identity of these events. However, if RB magnitude is not increased, this would suggest that RB reveals a capacity-limited aspect of conscious target perception independent of the central bottleneck. To compare the effect of response selection demands on $\mathrm{RB}$ relative to the $\mathrm{AB}$, we tested $\mathrm{RB}$ with and without an accompanying $\mathrm{AB}$ by manipulating target-distractor similarity, a manipulation that can eliminate the $\mathrm{AB}$ while leaving $\mathrm{RB}$ intact (Chun, 1997).

\section{METHOD}

\section{Subjects}

Six women and four men (mean age, 27 years), all members of the Vanderbilt University community, participated in the study. All subjects reported normal or corrected-to-normal visual acuity. Subjects received $\$ 10$ for participation.

\section{Design}

Four independent variables, all of which had two levels, were manipulated: distractor category (digits vs. computer symbols), target repetition (repeat vs. nonrepeat), response type (speeded vs. unspeeded), and lag (lag 3 [283.3 msec] and lag 8 [747.4 msec]). Following prior conventions (Chun, 1997; Jolicœur, 1998), target repetition and lag were manipulated within blocks of trials, whereas distractor category and response type were manipulated across blocks.

The principal dependent variable was the log 10 transformation of the percentage of trials in which both targets were reported accurately: $\log (\mathrm{T} 1 \& \mathrm{~T} 2)$. While the percentage of $\mathrm{T} 1$ correct trials where $\mathrm{T} 2$ is also accurate (T2|T1) is the measure typically adopted for assessing the $\mathrm{AB}$, this measure is not suitable for analyzing $\mathrm{RB}$, be- cause it is difficult to distinguish whether T1 or T2 was the stimulus perceived by the subject in repeat trials where only a single target is reported (Chun, 1997; but see Johnston et al., 2002, who demonstrated that, at least in speeded tasks, T2 is the stimulus missed on repeat trials). Hence, $\log (\mathrm{T} 2 \mid \mathrm{T} 1)$ data will only serve as a secondary, confirmatory analysis in the present study. Finally, the percentage of trials where T1 was correct (T1) is also reported to assess whether tradeoffs occurred between $\mathrm{T} 1$ accuracy and $\mathrm{AB}$ magnitude. A $\log 10$ transformation was performed on all accuracy measures because Schweickert (1985) has demonstrated that additivity between independent stages of processing, such as recognition (AB) and individuation (RB), can only be tested using log transformed accuracy data (see also Jiang \& Chun, 2001).

\section{Stimuli and Apparatus}

Stimuli were presented in Courier New font and at a viewing distance of approximately $57 \mathrm{~cm}$ subtended $1^{\circ}$ of visual angle. The targets were white letters (A and/or B) and the distractors were either white digits (0-9) or white computer symbols $(<,>,=, \#, \%, ?, /, *$, $@, \&)$. Each stimulus appeared centrally on a grey background for $93.4 \mathrm{msec}$. The experiment was programmed in MATLAB, using the Psychophysics Toolbox extension (Brainard, 1997; Pelli, 1997) and was conducted using a Dell Pentium IV PC computer with a $75-\mathrm{Hz}$ vertical refresh rate.

\section{Procedure}

Subjects initiated each trial by pressing the space bar. Trials began with a white fixation square for $500 \mathrm{msec}$, followed by the RSVP stream (containing ten distractors plus one or two targets). T1 appeared at serial position three and $\mathrm{T} 2$, when presented, occurred equally often at lags 3 and 8 . The response options for T1 were "A" or "B," and for T2 "A," "B," or "Nothing." Subjects made keypress responses to the targets with the right hand; the index finger was mapped to "A" ("K" key) and the middle finger to "B" ("L" key). Subjects entered "Nothing" by pressing the space bar. In speeded trials, subjects responded as soon as they observed the first target, and in unspeeded trials they reported T1 after the stream had concluded. The second target was always responded to at the end of the stream. In the unspeeded blocks, response screens for both T1 and T2 were shown that displayed "Target 1?" and "Target 2?" respectively, until subjects responded. In the speeded condition, only the $\mathrm{T} 2$ response screen was presented. Subjects were required to make their responses in the correct order.

Each test block was made up of 45 trials for each of the four target repetition $\times$ lag conditions and 20 catch trials (containing only $\mathrm{T} 1$ ), for a total of 200 trials per test block and, because there were four blocks of trials, 800 trials for the entire experiment. Half the subjects performed the two unspeeded-response blocks first, whereas the other half performed the speeded-response blocks first. The presentation order of distractor category blocks, within the pairs of response type blocks, was counterbalanced across subjects. The first five trials of each condition in each block were considered practice and were therefore not analyzed.

\section{RESULTS}

Figure 1 shows the mean percentage $\log (\mathrm{T} 1 \& \mathrm{~T} 2)$ accuracy as a function of distractor category, target repetition, and lag, plotted separately for the speeded and unspeeded conditions. In order to examine the effects of response type and distractor category independently on the $\mathrm{AB}$ (nonrepeat performance) and $\mathrm{RB}$ (nonrepeat performance - repeat performance), these phenomena were analyzed separately. In addition, we also conducted a further analysis to evaluate the influence of $\mathrm{T} 1$ reaction time (RT) on the two phenomena. 


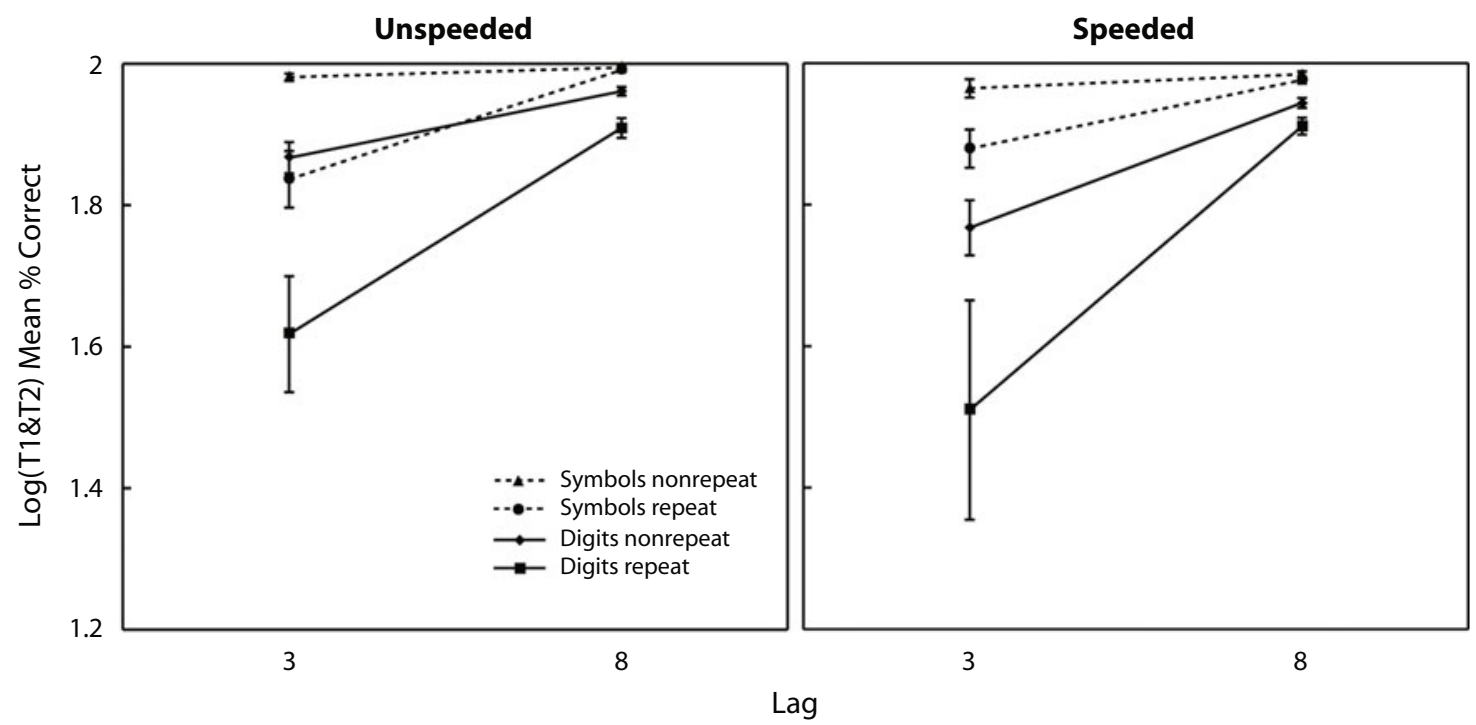

Figure 1. Mean percentage $\log (\mathrm{T} 1 \& \mathrm{~T} 2)$ accuracy as a function of distractor category, target repetition, and lag plotted separately for unspeeded and speeded trials. Error bars represent within-subjects standard error of the mean.

\section{AB}

The $\log (\mathrm{T} 1 \& \mathrm{~T} 2)$ accuracy data from nonrepeat trials were subjected to a $2 \times 2 \times 2$ repeated measures ANOVA with response type, distractor type, and lag as factors. A significant three-way interaction $[F(1,9)=6.7, p=$ .029] indicated that although there was a significant $\mathrm{AB}$ (performance lower at lag 3 than at lag 8) in both the unspeeded and speeded digit trials $(F \mathrm{~s}>41, p \mathrm{~s}<.0002)$, no significant $\mathrm{AB}$ was present in either the unspeeded or speeded symbol conditions $(F \mathrm{~s}<1.8, p \mathrm{~s}>.21)$. Most importantly, $\mathrm{AB}$ magnitude was larger in speeded digit trials than in unspeeded digit trials, as performance in the unspeeded condition was superior to that in the speeded condition at lag $3[F(1,9)=46, p=.0001]$, but not at lag $8(F=1.35, p=.27)$. These results replicate previous findings, demonstrating that the $\mathrm{AB}$ is strongly influenced by target-distractor discriminability (Chun, 1997), and that the second-target deficit is due, at least in part, to central processing limitations (Jolicœur, 1998). An identical pattern of results was obtained when $\log (\mathrm{T} 2 \mid \mathrm{T} 1)$ data were analyzed.

\section{RB}

The overall main effect of target repetition was significant $[F(1,9)=11.4, p<.0082]$, signaling the presence of RB (see Figure 1). Figure 2 plots RB magnitude (nonrepeat performance - repeat performance) as a function of distractor type, response type, and lag. The data were analyzed using a 2 (response type) $\times 2$ (distractor type) $\times 2$ (lag) repeated measures ANOVA. Like the AB, RB was temporally dependent; whereas there was significant $\mathrm{RB}$ at lag 3 , there was no significant repetition deficit at lag 8 $[F(1,9)=7.1, p=.0259]$. In addition, RB occurred not only for digit distractor trials but also for symbol distractor trials (the condition for which there was no significant $\mathrm{AB})$, as there was no significant effect of distractor type at lag $3(F=1.3, p=.27)$. Crucially, RB magnitude did not differ in speeded and unspeeded conditions, as evidenced by there being neither a main effect of response type, nor an interaction between response type and any of the other variables $(F \mathrm{~s}<1.03, p \mathrm{~s}>.32)$. These results demonstrate that, contrary to the $\mathrm{AB}$, making the $\mathrm{T} 1$ response online had no effect on RB.

\section{T1}

The $\log (\mathrm{T} 1)$ accuracy data were submitted to a $2 \times$ $2 \times 2$ repeated measures ANOVA with response type, distractor type, and lag as factors. Only data from nonrepeat trials were included in the analysis because, as previously discussed, it is difficult to assess individual target accuracy in unspeeded repeat trials (Chun, 1997). The results provide further evidence that speeding the T1 response increases the central processing demands of the T1 task, because performance was superior in unspeeded trials to that in speeded trials $[F(1,9)=22, p=.0011]$. In addition, the results demonstrated that symbol distractors interfered less with target processing than did digit distractors (Chun, 1997) because accuracy was higher in the symbol distractor condition than in the digit distractor condition $[F(1,9)=17.7, p=.0023]$. Importantly, the influence of distractor category and response type on $\log (\mathrm{T} 1)$ accuracy followed the pattern of results observed for the $\log (\mathrm{T} 1 \& \mathrm{~T} 2)$ data and the $\log (\mathrm{T} 2 \mid \mathrm{T} 1)$ data-namely, lower performance when the response was speeded and the distractors were digits. These results demonstrate that there were no trade-offs between $\mathrm{T} 1$ performance and $\mathrm{AB}$ magnitude.

\section{Influence of T1 RT on the AB and RB}

Examination of the $\log (\mathrm{T} 2 \mid \mathrm{T} 1)$ data as a function of $\mathrm{T} 1$ RT provides converging evidence that $\mathrm{RB}$, unlike the $\mathrm{AB}$, does not have its locus at the central bottleneck. ${ }^{1}$ When 


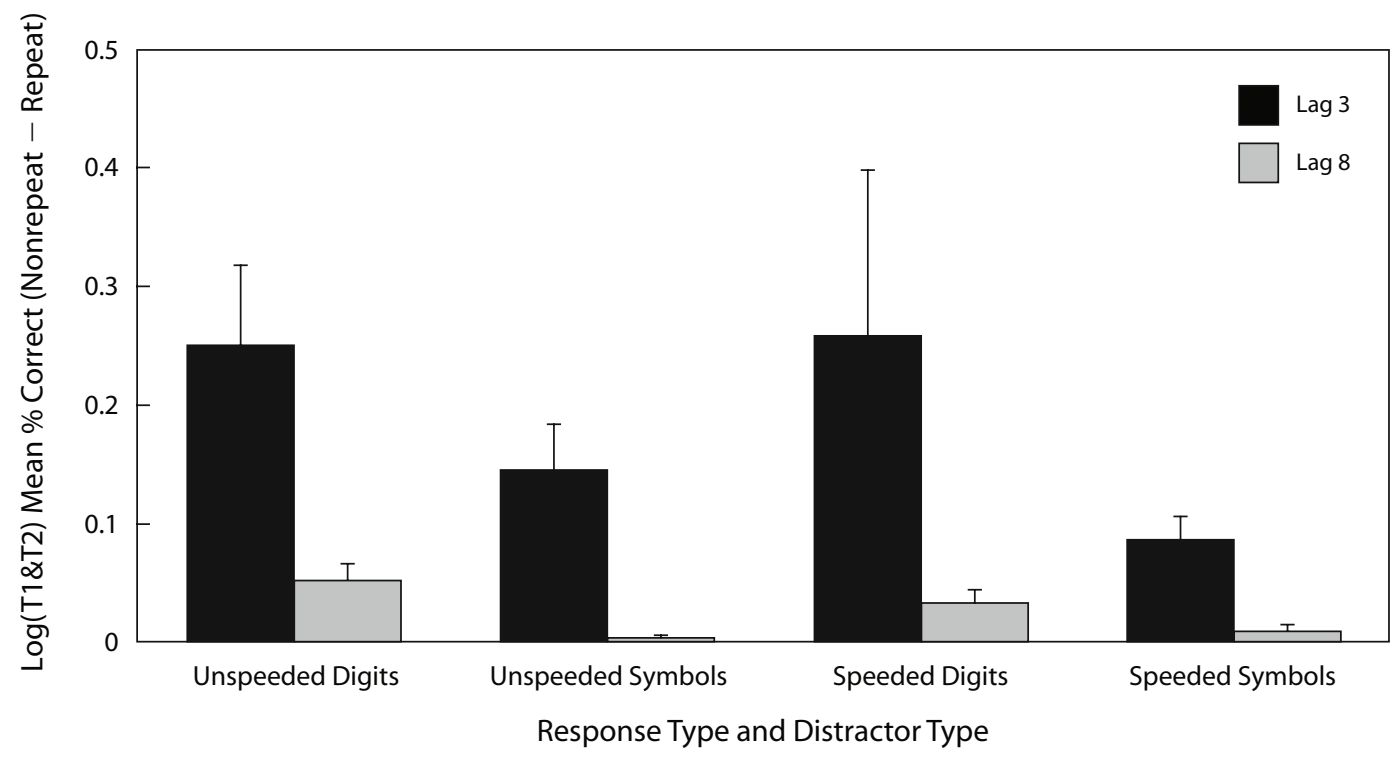

Figure 2. Mean percentage $\log (\mathrm{T} 1 \& \mathrm{~T} 2)$ accuracy difference between repeat and nonrepeat trials plotted as a function of distractor category, response type, and lag. Error bars represent within-subjects standard error of the mean.

$\mathrm{T} 1$ requires a speeded response in an $\mathrm{AB}$ paradigm, $\mathrm{T} 2$ accuracy is increasingly impaired with longer T1 RTs (Jolicœur, 1998). According to Jolicœur's central interference theory, the faster T1 is responded to, the less time T2's representation has to wait before it can access central processing resources. As a result, $\mathrm{AB}$ magnitude is reduced in trials where $\mathrm{T} 1$ is responded to quickly. We examined this hypothesis in our experiment by sorting T1 RT data into tertile bins in the nonrepeat digit condition (the condition that yielded maximal $\mathrm{AB}$ without accompanying $\mathrm{RB})$ and then assessing $\log (\mathrm{T} 2 \mid \mathrm{T} 1)$ accuracy performance as a function of this tertile variable (Figure 3 ). ${ }^{2}$ The data were entered into a $2(\mathrm{lag}) \times 3$ (T1 RT tertile) repeated measures ANOVA, which yielded a significant interaction $[F(2,12)=3.95, p=.048]$, demonstrating that the $\mathrm{AB}$ was larger in slower $\mathrm{T} 1$ (Tertile 3 ) trials than in faster T1 (Tertile 1) trials; the two conditions differed at lag 3 $[F(1,12)=13.3, p=.0034]$, but not at lag $8(F<1, p=$ .74). These results indicate that the magnitude of the $\mathrm{AB}$ is proportional to T1 RT, and are consistent with the idea that the $\mathrm{AB}$ is dependent on central resources. By contrast, the same analysis performed on the $\log (\mathrm{T} 2 \mid \mathrm{T} 1)$ data for the repeat symbol condition (Figure 3; the condition with maximal $\mathrm{RB}$ without accompanying $\mathrm{AB}$ ) showed no

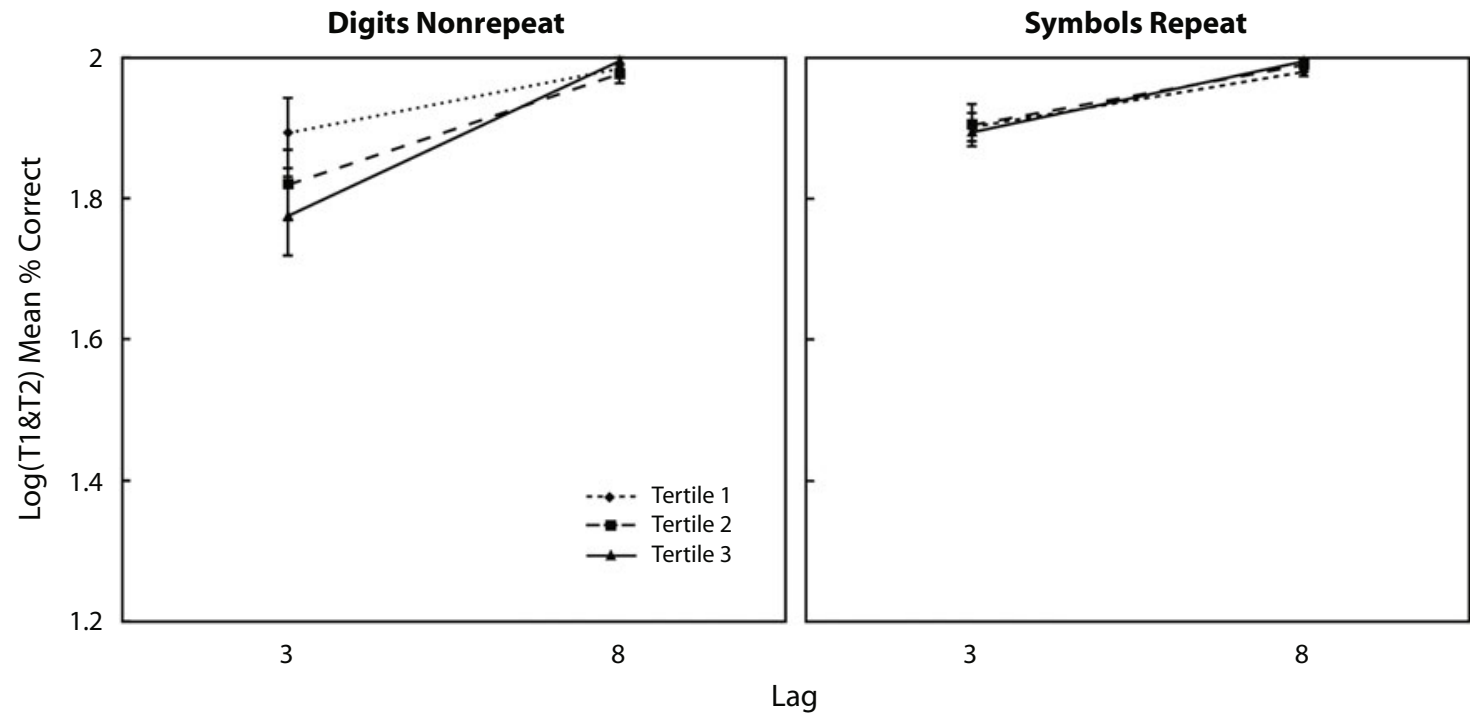

Figure 3. Mean percentage $\log (\mathrm{T} 2 \mid \mathrm{T} 1)$ accuracy as a function of T1 RT tertile and lag plotted separately for the nonrepeat digit and repeat symbol conditions. Error bars represent within-subjects standard error of the mean. 
difference in RB magnitude between the different T1 RT tertiles (interaction of lag and T1 RT tertile, $F<1, p=$ .88 ), further suggesting that RB does not have its locus at the central bottleneck.

\section{DISCUSSION}

Our study investigated whether RB, like the AB, reflects a central processing limitation. Specifically, by varying our methods, we investigated whether T1 required an online or offline response in a hybrid ABRB paradigm, and we thereby manipulated central response selection demands of the first target. The results clearly indicate that online response selection has strikingly different effects on the $\mathrm{AB}$ and $\mathrm{RB}$. The $\mathrm{AB}$ was increased when subjects made speeded responses to the first target, and the magnitude of this $\mathrm{AB}$ was proportional to $\mathrm{T} 1$ reaction time. These results are not only consistent with the central interference theory of the AB (Jolicœur, 1998), they also validate the use of this manipulation in assessing whether RB is similarly vulnerable to central processing demands. In stark contrast to the $\mathrm{AB}, \mathrm{RB}$ was not affected by online response selection, and its magnitude was independent of $\mathrm{T} 1$ reaction time. Taken together, these results suggest that, unlike the AB, $\mathrm{RB}$ is independent of central processing limitations.

Although we conclude that an increase in the response selection demands of the first target increases the magnitude of the $\mathrm{AB}$, we are not suggesting that manipulations of all stages of T1 information processing would necessarily influence this deficit. Indeed, whereas some studies have demonstrated that the magnitude of the $A B$ varies with manipulations of perceptual difficulty of T1 (Grandison, Ghirardelli, \& Egeth, 1997; Marois, Chun, \& Gore, 2004; Seiffert \& Di Lollo, 1997), others have not (McLaughlin, Shore, \& Klein, 2001; Shapiro, Raymond, \& Arnell, 1994; Ward et al., 1997). These inconsistent results are difficult to interpret, not only because of the methodological differences between these studies but also because, in unspeeded $A B$ tasks, it is difficult to assess which processing stages of the two targets might interfere with one another; subjects may delay particular processing stages until the end of the RSVP. With the present speeded task, we can assume that subjects executed the processing stages necessary for reporting the first target as quickly as possible. As a result, we can be confident that central stages involved in $\mathrm{T} 1$ processing overlapped with those performed on T2 and thereby led to the strong relationship we observed between $\mathrm{T} 1$ response selection demands and the $\mathrm{AB}$.

A final issue that warrants discussion concerns the stage of information processing at which RB takes place. As previously discussed, many theorists have suggested that the phenomenon occurs at perceptual stages of processing, when stimuli are encoded for conscious perception (e.g., Chun, 1997). However, others have argued for a later locus, such as memory retrieval (Fagot \& Pashler, 1995; for a review, see Neill, Neely, Hutchison, Kahan, $\&$ VerWys, 2002). Because the encoding of information into working memory (Jolicœur, 1998) and the retrieval of information from memory (Carrier \& Pashler, 1995) have both been shown to involve central processing, these processes are not likely to account for RB. Consistent with this conclusion, $\mathrm{RB}$ is still present when memory requirements are minimal (Johnston et al., 2002). Thus, together with these previous studies, our results are consistent with the view that RB is a perceptual deficit with a locus prior to the central bottleneck.

Regardless of the precise locus of RB, the present study clearly suggests that capacity limits to conscious visual perception in divided-attention paradigms are not all associated with processing limitations at a central bottleneck. Instead, our findings support the view that attentional capacity limits to visual awareness can occur at multiple stages of information processing.

\section{AUTHOR NOTE}

This research was supported by NSF Grant 0094992 and NIMH Grant R01 MH70776 to R.M. We thank Eric Ruthruff, Kimron Shapiro, and an anonymous reviewer for helpful comments on an earlier version of this manuscript. Correspondence concerning this article should be addressed to P. E. Dux, Department of Psychology, Vanderbilt University, 428 Wilson Hall, 111 21st Ave. So., Nashville, TN 37203 (e-mail: paul .dux@vanderbilt.edu).

\section{REFERENCES}

Arnell, K. M., \& Duncan, J. (2002). Shared and separate sources of dual-task cost in stimulus identification and response selection. Cognitive Psychology, 44, 105-147.

BRAINARD, D. H. (1997). The Psychophysics Toolbox. Spatial Vision, 10, 433-436.

CARRIER, L. M., \& PAShler, H. (1995). Attentional limits in memory retrieval. Journal of Experimental Psychology: Human Perception \& Performance, 21, 1339-1348.

Chun, M. M. (1997). Types and tokens in visual processing: A double dissociation between the attentional blink and repetition blindness. Journal of Experimental Psychology: Human Perception \& Performance, 23, 738-755.

CHUn, M. M., \& PotTer, M. C. (1995). A two-stage model for multiple target detection in rapid serial visual presentation. Journal of Experimental Psychology: Human Perception \& Performance, 21, 109-127.

FAGOT, C., \& PASHLER, H. (1995). Repetition blindness: Perception or memory failure? Journal of Experimental Psychology: Human Perception \& Performance, 21, 275-292.

Grandison, T. D., Ghirardelli, T. G., \& Egeth, H. E. (1997). Beyond similarity: Masking of the target is sufficient to cause the attentional blink. Perception \& Psychophysics, 59, 266-274.

JiANG, Y., \& CHUN, M. M. (2001). The influence of temporal selection on spatial selection and distractor interference: An attentional blink study. Journal of Experimental Psychology: Human Perception \& Performance, 27, 664-679.

Johnston, J. C., Hochhaus, L., \& Ruthruff, E. (2002). Repetition blindness has a perceptual locus: Evidence from online processing of targets in RSVP streams. Journal of Experimental Psychology: Human Perception \& Performance, 28, 477-489.

JoLICEUR, P. (1998). Modulation of the attentional blink by on-line response selection: Evidence from speeded and unspeeded Task deci- $^{-}$ sions. Memory \& Cognition, 26, 1014-1032.

Joliceur, P., Dell'AcQuA, R., \& CRebolder, J. M. (2001). The attentional blink bottleneck. In K. Shapiro (Ed.), The limits of attention: Temporal constraints in human information processing (pp. 82-99). New York: Oxford University Press.

KANWISHER, N. (1987). Repetition blindness: Type recognition without token individuation. Cognition, 27, 117-143.

LUCK, S. J., \& Vogel, E. K. (2001). Multiple sources of interference in dual-task performance: The cases of the attentional blink and the psychological refractory period. In K. Shapiro (Ed.), The limits of attention: Temporal constraints in human information processing (pp. 124-140). New York: Oxford University Press. 
Marois, R., Chun, M. M., \& Gore, J. C. (2004). A common parietofrontal network is recruited under both low visibility and high perceptual interference conditions. Journal of Neurophysiology, 92, 2985-2992.

Marois, R., \& Ivanoff, J. G. (2005). Capacity limits of information processing in the brain. Trends in Cognitive Sciences, 9, 296-305.

McLaughlin, E. N., Shore, D. I., \& Klein, R. M. (2001). The attentional blink is immune to masking-induced data limits. Quarterly Journal of Experimental Psychology, 54A, 169-196.

MORRIS, A. L., \& HARRIS, C. L. (2004). Repetition blindness: Out of sight or out of mind? Journal of Experimental Psychology: Human Perception \& Performance, 30, 913-922.

NeILl, W. T., Neely, J. H., Hutchison, K. A., Kahan, T. A., \& VerWys, C. A. (2002). Repetition blindness, forward and backward. Journal of Experimental Psychology: Human Perception \& Performance, 28, 137-149.

PASHLER, H. (1994). Dual-task interference in simple tasks: Data and theory. Psychological Bulletin, 116, 220-244.

PASHler, H. (1998). The psychology of attention. Cambridge, MA: MIT Press.

Pashler, H., \& Johnston, J. C. (1989). Chronometric evidence for central postponement in temporally overlapping tasks. Quarterly Journal of Experimental Psychology, 41A, 19-45.

PELLI, D. G. (1997). The VideoToolbox software for visual psychophysics: Transforming numbers into movies. Spatial Vision, 10, 437442.

Raymond, J. E., Shapiro, K. L., \& Arnell, K. M. (1992). Temporary suppression of visual processing in an RSVP task: An attentional blink? Journal of Experimental Psychology: Human Perception \& Performance, 18, 849-860.

Ruthruff, E., Miller, J., \& Lachmann, T. (1995). Does mental rotation require central mechanisms? Journal of Experimental Psychology: Human Perception \& Performance, 21, 552-570.

RUTHRuff, E., \& PASHLER, H. (2001). Perceptual and central interference in dual-task performance. In K. Shapiro (Ed.), The limits of attention: Temporal constraints in human information processing (pp. 100-123). New York: Oxford University Press.

SCHWEICKERT, R. (1985). Separable effects of factors on speed and accuracy: Memory scanning, lexical decision, and choice tasks. Psychological Bulletin, 97, 530-546.

SeIFFERT, A., \& Di LOLLO, V. (1997). Low-level masking in the attentional blink. Journal of Experimental Psychology: Human Perception \& Performance, 23, 1061-1073.

Sergent, C., \& Dehaene, S. (2004). Is consciousness a gradual phenomenon? Evidence for an all-or-none bifurcation during the attentional blink. Psychological Science, 15, 720-728.

Shapiro, K. L., RaYMOND, J. E., \& ARNEll, K. M. (1994). Attention to visual pattern information produces the attentional blink in rapid serial visual presentation. Journal of Experimental Psychology: Human Perception \& Performance, 20,357-371.

TELFORD, C. W. (1931). The refractory phase of voluntary and associative responses. Journal of Experimental Psychology, 14, 1-36.

Ward, R., Duncan, J., \& Shapiro, K. (1997). Effects of similarity, difficulty, and nontarget presentation on the time course of visual attention. Perception \& Psychophysics, 59, 593-600.

\section{NOTES}

1. Employing $\log (\mathrm{T} 2 \mid \mathrm{T} 1)$ accuracy to assess RB is valid in speeded trials, because Johnston et al. (2002) have demonstrated that under speeded repeat conditions $\mathrm{T} 2$ is the stimulus missed.

2. Three subjects were not included in this analysis as they displayed $\mathrm{ABs}$ in the speeded symbol condition. Hence, for these subjects RB effects could not be assessed independently from the AB. Importantly, RB magnitude (nonrepeat - repeat performance) was not influenced by T1 RT tertile $(F<1)$, even when these three subjects were included in the analysis.

(Manuscript received October 16, 2005; revision accepted for publication September 3, 2006.) 